\title{
BIFURCATION OF NONLINEAR EQUATIONS: I. STEADY STATE BIFURCATION*
}

\author{
TIAN $\mathrm{MA}^{\dagger}$ AND SHOUHONG WANG ${ }^{\ddagger}$
}

\begin{abstract}
We prove in this article some general steady state bifurcation theorem for a class of nonlinear eigenvalue problems, in the case where algebraic multiplicity of the eigenvalues of the linearized problem is even. These theorems provide an addition to the classical Krasnoselskii and Rabinowitz bifurcation theorems, which require the algebraic multiplicity of the eigenvalues is odd. For this purpose, we prove a spectral theorem for completely continuous fields, which can be considered as a generalized version of the classical Jordan matrix theorem and the Fredholm theorem for compact operators. An application to a system of second order elliptic equations is given as well.
\end{abstract}

Key words. steady state bifurcation from eigenvalues with even multiplicity, transcritical bifurcation, sadle-node bifurcation, spectral theorem

AMS subject classifications. 47J, 58E, 37L

1. Introduction. One of important topological tools for studying bifurcation of nonlinear partial differential equations is the classical Krasnoselskii and Rabinowitz bifurcation theorems; see among others L. Nirenberg [3]. The key assumption in these theorems is the oddness of the algebraic multiplicity of the eigenvalue of the linearized problem. To handle problems without the oddness assumption of the algebraic multiplicity of the eigenvalue, Krasnoselski studied bifurcations for potential operator equations [2]. Unfortunately, in many application problems, the eigenvalue of the linearized problem has even multiplicity, and the theorems are, in general, not applicable.

This article, which is Part I of a series of two articles, studies steady state bifurcation of nonlinear equations, and Part II is on dynamic bifurcations of time-dependent equations. The main objective of these articles is to establish corresponding theorems for the case where the eigenvalue has even multiplicity. Our main theorems include a general bifurcation theorem, Theorem 3.1, its global version, Theorem 3.2, and their special cases with more detailed count on the bifurcation branches, Theorems 4.2 and 5.1 .

These theorems are proved by calculating the topological degree of the equation, and the bifurcation is obtained when the degree changes as the parameter crosses a critical value. In the classical Rabinowitz and Krasnoselskii theorems, when the eigenvalue of the linear problem has odd multiplicity at the critical parameter, the degree, which are either +1 or -1 , changes sign as the parameter crosses the critical value, leading to bifurcation. This is essentially a linear theory as the degree is calculated, using the homotopy property of the degree, by calculating the degree of the linearized operator.

When the eigenvalue has even multiplicity, there is no change of the degree for the parameter on the two side of the critical value. The key idea in this article is

* Received May 10, 2004; accepted for publication September 28, 2004. The authors are grateful for the referees' insightful comments. The work was supported in part by the Office of Naval Research, by the National Science Foundation, and by the National Science Foundation of China.

${ }^{\dagger}$ Institute for Scientific Computing and Applied Mathematics \& Department of Mathematics, Indiana University, Bloomington, IN 47405, USA; Department of Mathematics, Sichuan University, Chengdu, P. R. China (tima@indiana.edu).

$¥$ Institute for Scientific Computing and Applied Mathematics \& Department of Mathematics, Indiana University, Bloomington, IN 47405, USA (showang@indiana.edu). 
based on the observation that for a class of nonlinearities, called $k$-th $(k \geq 2)$ order nondegenerate singularities, the degree at the critical parameter is even, creating the discrepancy of the degree, and leading to bifurcation. To carry out this idea, we need to introduce a spectral theorem, Theorem 2.3, which can be considered as a unified version of the Jordan theorem for matrices and the Fredholm theorem for compact operators.

The main results obtained can be easily applied to bifurcation problems in partial differential equtions from science and engineering. To demonstrate the applications, we present an example of a system of two second order elliptic equations. Bifurcation is obtained at the first eigenvalue, which has multiplicity 2.

This article is organized as follows. In Section 2, we introduce a spectral theorem, which is useful for the bifurcation study in the rest part of the article, together with genericity and density theorems for the spectrum of linear completely continuous fields, which may be useful in other contexts. Section 3 states and proves our general bifurcation theorem, and its global version. Two special cases are studied in Sections 4 and 5, and an example of applications is given in Section 6 .

2. Linear Completely Continuous Fields. In this section, we shall address some properties on the spectrum of linear completely continuous fields, some of which are useful for the bifurcation study addressed in this article.

2.1. Notations. Let $H$ and $H_{1}$ be two Hilbert spaces, and $H_{1} \hookrightarrow H$ be a dense and compact inclusion. A linear mapping $L=-A+B: H_{1} \rightarrow H$ is called a completely continuous field if $A: H_{1} \rightarrow H$ is a linear homeomorphism, and $B: H_{1} \rightarrow H$ is a linear compact operator.

A number $\lambda=\alpha+i \beta \in \mathbb{C}$ is called an eigenvalue of a linear operator $L: H_{1} \rightarrow H$ if there exist $x, y \in H_{1}$ with $x \neq 0$ such that

$$
L z=\lambda z \quad(z=x+i y),
$$

and the space

$$
E_{\lambda}=\left\{x, y \in H_{1} \quad \mid \quad(L-\lambda)^{n} z=0, \quad z=x+i y, \quad \text { for some } n \in \mathbb{N}\right\}
$$

is called the eigenspace of $L$ corresponding to $\lambda$, and $x, y \in E_{\lambda}$ are called eigenvectors of $L$.

Definition 2.1. A linear mapping $L^{*}: H_{1} \rightarrow H$ is called the conjugate operator of $L: H_{1} \rightarrow H$, if

$$
<L x, y>_{H}=<x, L^{*} y>_{H}, \quad \forall x, y \in H_{1} .
$$

A linear operator $L: H_{1} \rightarrow H$ is called symmetric if $L=L^{*}$.

Definition 2.2. A linear operator $L: H_{1} \rightarrow H$ has a complete eigenvalue sequence $\left\{\lambda_{k}\right\} \subset \mathbb{C}$ if each eigen-space $E_{k}$ corresponding to $\lambda_{k}$ is finite dimensional, and all eigenvectors of $L$ constitute a basis of $H$.

It is clear that eigenvalues $\lambda(a)$ of $L(a)=-A+a I+B$ are given by

$$
\lambda_{j}(a)=\lambda_{j}+a, \quad a \in \mathbb{R},
$$


where $I: H_{1} \rightarrow H$ is the inclusion mapping, $\lambda_{j}$ the eigenvalues of $L=-A+B$. Hence, for the eigenvalue problem, without loss of generality we always assume that $L$ has a compact inverse given by

$$
L^{-1}=-I \cdot A^{-1}\left(\mathrm{id}-B A^{-1}\right)^{-1}: \quad H \rightarrow H .
$$

Therefore, the eigenvalue problem of $L=-A+B$ can be equivalently written as the following form

$$
\left(L^{-1}-\beta_{k}\right)^{m} \psi_{k}=0, \quad \beta_{k}=\lambda_{k}^{-1}, \quad \text { for some } m \geq 1 .
$$

Let $H \otimes \mathbb{C}$ be the complexified space of $H$ given by

$$
H \otimes \mathbb{C}=\left\{\sum_{k} a_{k} u_{k} \quad \mid \quad u_{k} \in H, a_{k} \in \mathbb{C}\right\},
$$

with inner product

$$
<u, v>_{\hat{H}}=\left(<u_{1}, v_{1}>_{H}+<u_{2}, v_{2}>_{H}\right)+i\left(<u_{2}, v_{1}>_{H}-<u_{1} v_{2}>_{H}\right) .
$$

2.2. A spectral theorem. We begin with finite dimensional linear operators. Let $M$ be an $n \times n$ matrix, and $M^{*}$ its conjugate matrix. Let $\beta_{j}(j=1, \ldots, n)$ be all eigenvalues of $M$ (counting multiplicities). Vectors $\xi_{j} \in \mathbb{R}^{n}(1 \leq j \leq n)$ are called eigenvectors of $M$ if there exist $1 \leq k_{j} \leq m_{j}$ with $m_{j}$ being the multiplicity of $\beta_{j}$, such that

$$
\left(M-\beta_{j}\right)^{k_{j}} \xi_{j}=0
$$

when $\beta_{j}$ are real numbers, and

$$
\left\{\begin{array}{l}
\left(M-\beta_{j}\right)^{k_{j}}\left(\xi_{j}+i \xi_{j+1}\right)=0, \\
\left(M-\beta_{j+1}\right)^{k_{j}}\left(\xi_{j}-i \xi_{j+1}\right)=0,
\end{array}\right.
$$

when $\beta_{j}=\bar{\beta}_{j+1}$ are complex numbers, with $\beta_{j+1}$ being the complex conjugate of $\beta_{j}$.

Let $\xi_{j}=\left(\xi_{j 1}, \cdots, \xi_{j n}\right)^{t}$ be eigenvectors of $M$ such that

$$
M P=P J,
$$

where $J$ is the Jordan form of $M$, and

$$
P=\left(\begin{array}{cccc}
\xi_{11} & \xi_{21} & \cdots & \xi_{n 1} \\
\xi_{12} & \xi_{22} & \cdots & \xi_{n 2} \\
\vdots & \vdots & & \vdots \\
\xi_{1 n} & \xi_{2 n} & \cdots & \xi_{n n}
\end{array}\right)
$$

Then it is easy to see that

$$
M^{t}\left(P^{-1}\right)^{t}=\left(P^{-1}\right)^{t} J^{t},
$$

where $J^{t}$ is the transpose of $J$, which is also the Jordan form of conjugate matrix (transpose) $M^{*}=M^{t}$. Hence if we set

$$
\left(P^{-1}\right)^{t}=\left(\widetilde{\xi}_{1}, \cdots, \widetilde{\xi}_{n}\right),
$$


then $\widetilde{\xi}_{1}, \cdots, \widetilde{\xi}_{n}$ are eigenvectors of $M^{*}$,

$$
P^{-1}=\left(\begin{array}{c}
\widetilde{\xi}_{1}^{t} \\
\vdots \\
\widetilde{\xi}_{n}^{t}
\end{array}\right)
$$

and

$$
I=P^{-1} P=\left(\begin{array}{c}
\widetilde{\xi}_{1}^{t} \\
\vdots \\
\widetilde{\xi}_{n}^{t}
\end{array}\right)\left(\begin{array}{lll}
\xi_{1} & \cdots, & \xi_{n}
\end{array}\right)=\left(\begin{array}{cccc}
\widetilde{\xi}_{1}^{t} \xi_{1} & \widetilde{\xi}_{1}^{t} \xi_{2} & \cdots & \widetilde{\xi}_{1}^{t} \xi_{n} \\
\widetilde{\xi}_{2}^{t} \xi_{1} & \widetilde{\xi}_{2}^{t} \xi_{2} & \cdots & \widetilde{\xi}_{2}^{t} \xi_{n} \\
\vdots & \vdots & & \vdots \\
\widetilde{\xi}_{n}^{t} \xi_{1} & \widetilde{\xi}_{n}^{t} \xi_{2} & \cdots & \widetilde{\xi}_{n}^{t} \xi_{n}
\end{array}\right)
$$

We note that equations (2.6) and (2.8) are equivalent to the classical Fredholm alternative theorem. Therefore, from (2.10) we get a theorem as follows, which is considered as a unified version of the Fredholm alternative theorem and the Jordan theorem.

Theorem 2.3. Let $L=-A+B: H_{1} \rightarrow H$ be a linear completely continuous field. Then the following assertions hold true.

(1) If $\left\{\lambda_{k} \mid k \geq 1\right\} \subset \mathbb{C}$ are eigenvalues of $L$, then we can take the eigenvectors of $\left\{\phi_{k}\right\} \subset H_{1}$ of $L$ and eigenvectors $\left\{\phi_{k}^{*}\right\} \subset H_{1}$ of the conjugate operator $L^{*}$ such that

$$
<\phi_{i}, \phi_{j}^{*}>_{H}=\delta_{i j}, \quad \delta_{i j} \text { the Kronecker symbol. }
$$

(2) $H$ can be decomposed into the following direct sum

$$
\left\{\begin{array}{l}
H=E_{1} \oplus E_{2}, \\
E_{1}=\text { the closure of } \operatorname{span}\left\{\phi_{k} \mid k \geq 1\right\} \text { in } H, \\
E_{2}=\left\{v \in H \mid<v, \phi_{k}^{*}>_{H}=0, \forall k \geq 1\right\} .
\end{array}\right.
$$

(3) $E_{1}$ and $E_{2}$ are invariant spaces of $L^{-1}$, and

$$
\left\|L^{-n} v\right\|_{H}^{1 / n} \rightarrow 0 \quad \text { as } n \rightarrow \infty, \forall v \in E_{2} .
$$

(4) Let $\beta_{1}, \cdots, \beta_{k} \in \mathbb{C}$ be eigenvalues of $L^{-1}$ (counting multiplicities) in the order of $\left|\beta_{1}\right| \geq\left|\beta_{2}\right| \geq \cdots \geq\left|\beta_{k}\right|, \psi_{1}^{*}, \cdots, \psi_{k}^{*} \in H \otimes \mathbb{C}$ be the corresponding eigenvectors of $\left(L^{-1}\right)^{*}=\left(L^{*}\right)^{-1}$, and let $E_{k}^{*}=\operatorname{span}\left\{\psi_{1}^{*}, \cdots, \psi_{k}^{*}\right\}\left(E_{k}^{*}=\emptyset\right.$ as $k=0)$. If

$$
\rho_{k+1}=\left.\sup _{\substack{u \in H \\\|u\|=1, u \perp E_{k}^{*}}} \lim _{n \rightarrow \infty}\left|<L^{-n} u, u\right\rangle_{\hat{H}}\right|^{1 / n}>0,
$$

then there is an eigenvalue $\beta_{k+1} \in \mathbb{C}$ of $L^{-1}$ with $\left|\beta_{k+1}\right|=\rho_{k+1}$, and $\left|\beta_{k+1}\right| \leqslant$ $\left|\beta_{k}\right|$. 
REMARK 2.4. By (2.11) and (2.12), for any $u \in H$, we have the generalized Fourier expansion

$$
u=\sum_{k} u_{k} \phi_{k}+v, v \in E_{2}, u_{k}=<u, \phi_{k}^{*}>_{H} .
$$

In particular, if the operator $L=-A+B: H_{1} \rightarrow H$ has a complete eigenvalue sequence $\lambda_{k} \subset \mathbb{C}$ with eigenvector sequence $\left\{\phi_{k}\right\} \subset H_{1}$, then we have the following complete Fourier expansion

$$
u=\sum_{k=1}^{\infty} u_{k} \phi_{k}, \quad u_{k}=<u, \phi_{k}^{*}>_{H} .
$$

Remark 2.5. Let $X$ be a Banach space, $X^{*}$ its dual space, and $B: X \rightarrow X$ a compact linear operator with its conjugate operator $B^{*}: X^{*} \rightarrow X^{*}$. Then the above spectral theorem holds true as well for the eigenvalue problem $B x=\lambda x$. Assertions (1) and (2) in Theorem 2.3 can be considered as a generalized version of the classical Fredholm Alternative theorem and Jordan theorem.

Proof of Theorem 2.3. We proceed in several steps as follows.

SteP 1. It is known that the space $H$ can be decomposed into direct sums of invariant spaces of $L^{-1}$ as follows

$$
\left\{\begin{array}{l}
H=E_{1} \oplus E_{2}, \quad H=E_{1}^{*} \oplus E_{2}^{*}, \\
E_{1}=\text { the closure of span }\left\{\phi_{k} \mid k \geq 1\right\} \text { in } H, \\
E_{1}^{*}=\text { the closure of span }\left\{\phi_{k}^{*} \mid k \geq 1\right\} \text { in } H .
\end{array}\right.
$$

By the spectral radius theorem, we have

$$
\left\{\begin{array}{l}
\left\|L^{-n} v\right\|_{H}^{1 / n} \rightarrow 0 \quad \text { as } \quad n \rightarrow \infty, \forall v \in E_{2}, \\
\left\|L^{*-n} v\right\|_{H}^{1 / n} \rightarrow 0 \text { as } \quad n \rightarrow \infty, \forall v \in E_{2}^{*} .
\end{array}\right.
$$

Assertion (3) follows.

StEP 2. Let $\left\{\xi_{1}, \cdots, \xi_{m}\right\} \subset H_{1} \otimes \mathbb{C}$ be the eigenvectors of $L$ corresponding to an eigenvalue $\lambda \in \mathbb{C}$, and $\left\{\eta_{1}^{*}, \cdots, \eta_{M}^{*}\right\}$ be the eigenvectors of $L^{*}$ corresponding to an eigenvalue $\rho \in \mathbb{C}$. Set

$$
\xi_{l}=\xi_{l 1}+i \xi_{l 2}, \quad \eta_{j}^{*}=\eta_{j 1}^{*}+i \eta_{j 2}^{*}, \quad 1 \leq l \leq m, 1 \leq j \leq M .
$$

We shall prove that if $\lambda \neq \rho$,

$$
\begin{aligned}
& <\xi_{l k}, \eta_{j r}^{*}>_{H}=0, \forall k, r=1,2,1 \leq l \leq m, 1 \leq j \leq M, \\
& <v, \phi_{k}^{*}>_{H}=0,<v^{*}, \phi_{k}>_{H}=0, \forall k \geq 1, v \in E_{2}, v^{*} \in E_{2}^{*}, \\
& <\xi_{l k}, \xi_{j r}^{*}>_{H}=\delta_{l j} \cdot \delta_{k r} .
\end{aligned}
$$

For simplicity, we consider only the case where $m=M=2$, and the geometric multiplicities of $\lambda$ and $\rho$ are one; while the general case can be proved in the same fashion. Then we have

$$
\begin{aligned}
& L^{-1} \xi_{1}=\lambda^{-1} \xi_{1}, \quad L^{-1} \xi_{2}=\lambda^{-1} \xi_{2}+\epsilon \xi_{1}, \quad \epsilon \in \mathbb{R}, \\
& L^{*-1} \eta_{2}^{*}=\rho^{-1} \eta_{2}^{*}, \quad L^{*-1} \eta_{1}^{*}=\rho^{-1} \eta_{1}^{*}+\gamma \eta_{2}^{*}, \quad \gamma \in \mathbb{R} .
\end{aligned}
$$


As $\lambda \neq \bar{\rho}$, it follows from (2.20) and (2.21) that

$$
\begin{aligned}
<\xi_{1}, \eta_{2}^{*}>_{\hat{H}} & =<\xi_{11}+i \xi_{12}, \eta_{21}^{*}-i \eta_{22}^{*}>_{H} \\
& =\lambda<L^{-1} \xi_{1}, \eta_{2}^{*}>_{\hat{H}} \\
& =\lambda<\xi_{1}, L^{*-1} \eta_{2}^{*}>_{\hat{H}} \\
& =\lambda \bar{\rho}^{-1}<\xi_{1}, \eta_{2}^{*}>_{\hat{H}} \\
& =0 \\
<\xi_{1}, \bar{\eta}_{2}^{*}>_{\hat{H}} & =\lambda \rho^{-1}<\xi_{1}, \bar{\eta}_{2}^{*}>_{\hat{H}} \\
& =0
\end{aligned}
$$

which imply that

$$
<\xi_{1 k}, \eta_{2 r}^{*}>_{H}=0, k, r=1,2 .
$$

Similarly, we can induce from (2.20) and (2.21) that

$$
<\xi_{i}, \eta_{j}^{*}>_{\hat{H}}=0,<\xi_{i}, \bar{\eta}_{j}^{*}>_{\hat{H}}=0, \quad 1 \leq i, j \leq 2 .
$$

Thus, we have proved (2.17), and (2.18) can be obtained in the same fashion from (2.3) and (2.16).

STEP 3. Now, we shall prove (2.19) when $\lambda=\bar{\rho}=$ complex number; while the case where $\lambda=\rho=$ real number can be proved in the same fashion. Let $\lambda=\alpha+i \beta$, $\eta_{k}^{*}=\xi_{k}^{*}=\xi_{k 1}^{*}+i \xi_{k 2}^{*}$. From (2.20) and (2.21) with $\gamma=\epsilon$, we get

$$
\mathcal{L} \xi=\xi J
$$

$$
\mathcal{L}^{*} \xi^{*}=\xi^{*} J^{*}
$$

Here $\mathcal{L}$ is an infinite matrix induced by $L^{-1}$ under a canonical orthogonal basis $\left\{h_{k}\right\}$ of $H$, 


$$
\begin{aligned}
\mathcal{L} & =\left(\begin{array}{lll}
a_{11} & a_{12} & \cdots \\
a_{21} & a_{22} & \cdots \\
\cdots & \cdots & \cdots
\end{array}\right), \\
\mathcal{L}^{*} & =\left(a_{i j}\right)^{t}=\left(\begin{array}{ccc}
a_{11} & a_{21} & \cdots \\
a_{12} & a_{22} & \cdots \\
\cdots & \cdots & \cdots
\end{array}\right), \\
\xi & =\left(\begin{array}{cccc}
\xi_{11}^{1} & \xi_{12}^{1} & \xi_{21}^{1} & \xi_{22}^{1} \\
\xi_{11}^{2} & \xi_{12}^{2} & \xi_{21}^{2} & \xi_{22}^{2} \\
\vdots & \vdots & \vdots & \vdots
\end{array}\right), \\
\xi^{*} & =\left(\begin{array}{cccc}
\xi_{11}^{1 *} & \xi_{12}^{1 *} & \xi_{21}^{1 *} & \xi_{22}^{1 *} \\
\xi_{11}^{2 *} & \xi_{12}^{2 *} & \xi_{21}^{2 *} & \xi_{22}^{2 *} \\
\vdots & \vdots & \vdots & \vdots
\end{array}\right), \\
J & =\left(\begin{array}{cccc}
\alpha & -\beta & \epsilon & 0 \\
\beta & \alpha & 0 & \epsilon \\
0 & 0 & \alpha & -\beta \\
0 & 0 & \beta & \alpha
\end{array}\right), \\
J^{*} & =J^{t}=\left(\begin{array}{cccc}
\alpha & \beta & 0 & 0 \\
-\beta & \alpha & 0 & 0 \\
\epsilon & 0 & \alpha & \beta \\
0 & \epsilon & -\beta & \alpha
\end{array}\right),
\end{aligned}
$$

where

$$
\begin{aligned}
& a_{i j}=<L^{-1} h_{j}, h_{i}>_{H}, \\
& \xi_{k r}^{j}=<\xi_{k r}, h_{j}>_{H}, \\
& \xi_{k r}^{j *}=<\xi_{k r}^{*}, h_{j}>_{H} .
\end{aligned}
$$

It is clear that (2.19) is equivalent to

$$
\xi^{* t} \cdot \xi=I,
$$

where $I$ is the $4 \times 4$ identity matrix.

It is known that for each pair of indices $(k, r)$ there exist $\zeta_{k r}^{*} \in H$ and $\zeta_{k r} \in H$ which are unique as the functionals on $H$, such that

$$
\left\{\begin{aligned}
& <\zeta_{k r}^{*}, \xi_{k r}>_{H}=1, \\
& <\zeta_{k r}^{*}, \phi_{j}>_{H}=0, \quad \forall \phi_{j} \neq \xi_{k r} \\
& <\xi_{k r}^{*}, v>_{H}=0, \quad \forall v \in E_{2}
\end{aligned}\right.
$$

and

$$
\left\{\begin{array}{l}
<\zeta_{k r}, \xi_{k r}^{*}>_{H}=1, \\
<\zeta_{k r}, \phi_{j}^{*}>_{H}=0, \quad \forall \phi_{j}^{*} \neq \xi_{k r}^{*} \\
<\xi_{k r}, v^{*}>_{H}=0 \quad \forall v^{*} \in E_{2}^{*} .
\end{array}\right.
$$


Therefore, the matrices

$$
\begin{aligned}
\zeta^{* 1} & =\left(\begin{array}{lll}
\zeta_{11}^{* 1} & \zeta_{11}^{* 2} & \ldots \\
\zeta_{12}^{* 1} & \zeta_{12}^{* 2} & \ldots \\
\zeta_{21}^{* 1} & \zeta_{21}^{* 2} & \ldots \\
\zeta_{22}^{* 1} & \zeta_{22}^{* 2} & \ldots
\end{array}\right), \zeta_{k r}^{* j}=<\zeta_{k r}^{*}, h_{j}>_{H}, \\
\zeta^{1} & =\left(\begin{array}{lll}
\zeta_{11}^{1} & \zeta_{11}^{2} & \ldots \\
\zeta_{12}^{1} & \zeta_{12}^{2} & \cdots \\
\zeta_{21}^{1} & \zeta_{21}^{2} & \ldots \\
\zeta_{22}^{1} & \zeta_{22}^{2} & \ldots
\end{array}\right), \zeta_{k r}^{j}=<\zeta_{k r}, h_{j}>_{H},
\end{aligned}
$$

are the left inverses of $\xi$ and $\xi^{*}$ respectively. Hence, it follows from (2.22) and (2.23) that

$$
\begin{aligned}
& \zeta^{*} \mathcal{L} \xi=J, \\
& \zeta \mathcal{L}^{*} \xi^{*}=J^{*} .
\end{aligned}
$$

The transpose of (2.28) implies that

$$
\left(\xi^{*}\right)^{t} \mathcal{L} \zeta^{t}=J
$$

Since $\zeta^{*}$ and $\zeta$ satisfy (2.25) and (2.26) respectively, by

$$
\zeta^{*}=\xi^{* t}, \quad \zeta=\xi^{t},
$$

which yield (2.24). Thus, Assertions (1) and (2) are derived from (2.15) and (2.17)-(2.19)

Step 4. Proof of Assertion (4). It is clear that the numbers defined by (2.13) satisfy $0 \leq \rho \leq\left\|L^{-1}\right\|$. By the spectral radius theorem, if $\rho>0$ then $L^{-1}$ must have at least an eigenvalue $\beta \in \mathbb{C}$. Let $\beta_{j} \in \mathbb{C}(j \geq 1)$ be the eigenvalues of $L^{-1}$ in the order

$$
\left|\beta_{1}\right| \geq\left|\beta_{2}\right| \geq \cdots \geq\left|\beta_{j}\right| \geq\left|\beta_{j+1}\right| \geq \cdots
$$

and $\left\{\psi_{j}\right\} \subset H \otimes \mathbb{C}$ be the eigenvectors of $L^{-1}$ (counting multiplicities), then by Assertions 1)-3), for any $u \in H \otimes \mathbb{C}$ with $u \perp E_{k}^{*}(k \geq 0), u$ can be expressed as

$$
\begin{aligned}
& u=\sum_{j=k+1} u_{j} \psi_{j}+v, \quad u_{j}=<u, \psi_{j}^{*}>_{\hat{H}} \in \mathbb{C}, \\
& \lim _{n \rightarrow \infty}\left\|L^{-n} v\right\|^{1 / n}=0 .
\end{aligned}
$$

For simplicity, we assume that the eigenvalues $\beta_{j}$ are simple, and other case can be proved in the same fashion. Then we have

$$
\begin{aligned}
\left|<L^{-n} u, u>_{\hat{H}}\right| & =\left|<\sum_{j \geq k+1} \beta_{j}^{n} u_{j} \psi_{j}+L^{-n} v, \sum_{i \geq k+1} \bar{u}_{i} \bar{\psi}_{i}+\bar{v}>_{H}\right| \\
& =\left|\sum_{j \geq k+1} a_{j} \beta_{j}^{n}+<L^{-n} v, \bar{u}>_{H}\right|,
\end{aligned}
$$

where

$$
a_{j}=\sum_{i \geq k+1} u_{j} \bar{u}_{i}<\psi_{j}, \bar{\psi}_{i}>_{H}+u_{j}<\psi_{j}, \bar{v}>_{H} .
$$

Then by (2.30) and (2.31), we obtain that $\left|\beta_{k+1}\right|=\rho_{k+1}$.

The proof of the theorem is complete. $\square$ 
2.3. Density of complete spectrum. The spectral completion is a very interesting and difficult problem. It is known that if a completely continuous field $L: H_{1} \rightarrow H$ is symmetric sectorial operator, then $L$ has a complete eigenvalue sequence. But, for general sectorial operators, we don't know if the spectral completion theorem holds true. For this purpose, we prove in this section a density theorem of complete spectrum.

To proceed, we first recall that $A: H_{1} \rightarrow H$ is a sectorial operator if the sector

$$
S_{a, \phi}=\left\{\lambda \in \mathbb{C}|\phi<| \arg (\lambda-a) \mid \leq \pi, 0<\phi \leq \frac{\pi}{2}\right\}
$$

for some $a \in R$ is in the resolvent set of $A$, and

$$
\left\|(A-\lambda I)^{-1}\right\| \leq \frac{M}{|\lambda-a|}, \quad \forall \lambda \in S_{a, \phi},
$$

for some $M \geq 1$. Then we can define fractional Hilbert spaces $H_{\alpha}=D\left(A^{\alpha}\right)$ with induced norm denoted by $\|u\|_{\alpha}$. We know that $H_{0}=H, H_{\alpha}=H_{1}$ as $\alpha=1$, and $H_{\alpha} \hookrightarrow H_{\beta}$ is a compact inclusion for all $\alpha>\beta$.

Now consider a linear completely continuous field $L=-A+B: H_{1} \rightarrow H$ such that

$$
\left\{\begin{array}{l}
A: H_{1} \rightarrow H \text { is sectorial, } \\
A \text { has a complete eigenvalue sequence }\left\{\lambda_{k}\right\} \\
\operatorname{Re} \lambda_{k}>0, \quad \forall k \geq 1
\end{array}\right.
$$

and the linear operator $B: H_{1} \rightarrow H$ satisfies

$$
B: H_{\gamma} \rightarrow H \text { is bounded for some } \gamma<1 .
$$

By (2.32) and (2.33), we know that the operator $L=-A+B: H_{1} \rightarrow H$ is also a sectorial operator, and if $L$ has no eigenvalues with nonnegative real parts, then we can define a fractional power operator $L^{\alpha}(\alpha \in \mathbb{R})$ with domain $D\left(L^{\alpha}\right)=D\left(A^{\alpha}\right)=H_{\alpha}$, and the norm of $H_{\alpha}$ is equivalent to $\left\|L^{\alpha} u\right\|_{0}$ for $u \in H_{\alpha}$.

Let

$$
\begin{aligned}
& \mathcal{S}\left(H_{1}, H\right)=\left\{-A+B: H_{1} \rightarrow H \text { satisfies }(2.32) \text { and }(2.33)\right\} \\
& \mathcal{S}_{A}\left(H_{1}, H\right)=\left\{L=-A+B \in \mathcal{S}\left(H_{1}, H\right) \mid A: H_{1} \rightarrow H \text { is fixed }\right\} .
\end{aligned}
$$

Then we have the following density theorem for complete spectrum.

TheOREM 2.6. There exists a dense set $D \subset \mathcal{S}\left(H_{1}, H\right)\left(\right.$ resp. $\left.D \subset \mathcal{S}_{A}\left(H_{1}, H\right)\right)$ such that each operator $L \in D$ has a complete eigenvalue sequence.

REMARK 2.7. In fact, it is reasonable to conjecture that each $L \in \mathcal{S}\left(H_{1}, H\right)$ satisfying (2.32) and (2.33) has a complete eigenvalue sequence.

Proof of Theorem 2.6. Let $L=-A+B \in \mathcal{S}\left(H_{1}, H\right)$, and $\left\{\phi_{k}\right\} \subset H_{1}$ be eigenvectors of $A$. By (2.14), for any $u \in H, u=\sum_{i=0}^{\infty} u_{i} \phi_{i}$. Let $P_{n}: H \rightarrow H$ be the canonical projection defined by

$$
P_{n} u=\sum_{i=1}^{n} u_{i} \phi_{i}
$$


As $P_{n} B: H_{1} \rightarrow H$ is finite rank, the operator $L_{n}=-A+P_{n} B$ has a complete eigenvalue sequence for any $n \in \mathbb{N}$. In addition, $B: H_{1} \rightarrow H$ is compact, we have

$$
\lim _{n \rightarrow \infty}\left\|B-P_{n} B\right\|_{\mathcal{S}\left(H_{1}, H\right)}=0 .
$$

Thus we obtain

$$
\lim _{n \rightarrow \infty}\left\|L-L_{n}\right\|_{\mathcal{S}\left(H_{1}, H\right)}=\left\|B-P_{n} B\right\|_{\mathcal{S}\left(H_{1}, H\right)}=0 .
$$

Therefore, the proof of the theorem is complete.

2.4. Genericity of simple eigenvalues. An eigenvalue $\lambda \in \mathbb{C}$ of $L: H_{1} \rightarrow H$ is called simple if the eigenspace $E_{\lambda}$ of $L$ has dimension

$$
\operatorname{dim} E_{\lambda}= \begin{cases}1 & \text { as } \lambda=\text { real, } \\ 2 & \text { as } \lambda=\text { complex. }\end{cases}
$$

Let the eigenvalues $\left\{\lambda_{k}\right\}$ of a linear operator $L \in \mathcal{S}\left(H_{1}, H\right)$ be given in the following order

$$
\left|\lambda_{1}\right| \leq\left|\lambda_{2}\right| \leq \cdots\left|\lambda_{k}\right| \leq\left|\lambda_{k+1}\right| \leq \cdots .
$$

The following is the genericity theorem for simple eigenvalues.

THEOREM 2.8. For any integer $m \geq 1$, there is an open and dense set $\mathcal{O} \subset$ $\mathcal{S}\left(H_{1}, H\right)$ (resp. $\mathcal{O} \subset \mathcal{S}_{A}\left(H_{1}, H\right)$ ), such that each $L \in \mathcal{O}$ has at least $m$ eigenvalues, and the first eigenvalues $\left\{\lambda_{j} \mid j=1, \cdots, m\right\}$ are simple.

Proof. By the classical theory of linear operators, each isolated eigenvalue depends continuously on the operators; see [1]. Therefore, by Theorem 2.6, we obtain that the set $\widetilde{\mathcal{O}}$ of all linear operators having at least $m$ eigenvalues is open and dense in $\mathcal{S}\left(H_{1}, H\right)$ (resp. in $\left.\mathcal{S}_{A}\left(H_{1}, H\right)\right)$.

Let $\mathcal{O} \subset \widetilde{\mathcal{O}}$ be the set of all linear operators whose first $m$ eigenvalues are simple. Obviously, $\mathcal{O}$ is an open set. We shall prove that for any $L=-A+B \in \widetilde{\mathcal{O}}$, there is a sequence $L_{n}=-A+B_{n} \in \mathcal{O}$, which converges to $L$ in $\mathcal{S}\left(H_{1}, H\right)$.

Let $\left\{\phi_{k}\right\}$ be the eigenvalues of $L$. Without loss of generality, we assume that the first eigenvalues of $L$ have multiplicity two, i.e. $\lambda_{1}=\lambda_{2}$, and

$$
L \phi_{1}=\lambda_{1} \phi_{1}, \quad L \phi_{2}=\lambda_{1} \phi_{2}+\phi_{1} .
$$

By (2.14), for any $u \in H, u=\sum_{k} u_{k} \phi_{k}+v, v \in E_{2}$. We define a linear operator $T_{n}: H_{1} \rightarrow H$ by

$$
T_{n} u=\frac{1}{n} u_{1} \phi_{1} .
$$

Them we infer from (2.34) that the first two eigenvalues $\lambda_{1}$ and $\lambda_{1}+\frac{1}{n}$ of the operator $L_{n}=-A+B+T_{n}$ are simple, i.e.

$$
\begin{aligned}
& L_{n}\left(\phi_{2}-n \phi_{1}\right)=\lambda_{1}\left(\phi_{2}-n \phi_{1}\right), \\
& L_{n} \phi_{1}=\left(\lambda_{1}+\frac{1}{n}\right) \phi_{1} .
\end{aligned}
$$

Obviously, $B+T_{n}$ satisfies (2.33), and $L_{n} \rightarrow L$ in $\mathcal{S}\left(H_{1}, H\right)$ as $n \rightarrow \infty$. We can prove in the same fashion that for each $n \in \mathbb{N}$ there exists an $B_{n}: H_{1} \rightarrow H$ such that (2.33) holds true, $B_{n} \rightarrow B$ in $\mathcal{S}\left(H_{1}, H\right)$ as $n \rightarrow \infty$, and the first $m$ eigenvalues of $L_{n}=-A+B_{n}$ are simple.

The proof is complete. 
3. Steady State Bifurcation from Higher-Order Non-degenerate Singularities. As mentioned in the Introduction, the Krasnoselskii bifurcation theorem asserts that steady state bifurcation occurs at an eigenvalue with odd multiplicity, and there is no general result for bifurcations at an eigenvalue with even multiplicity. In this section, we shall give some bifurcation theorems at such eigenvalues with even multiplicities.

3.1. Main theorems. Consider a parameter family of nonlinear operator equations

$$
L_{\lambda} u+G(u, \lambda)=0,
$$

where $u \in H$ is the unknown function, $\lambda \in \mathbb{R}$ the parameter, for each $\lambda, L_{\lambda}: H_{1} \rightarrow H$ is a completely continuous field, and $G(\cdot, \lambda): H_{1} \rightarrow H$ is a $C^{r}(r \geq 1)$ mapping, depending continuously on the parameter $\lambda \in \mathbb{R}$. We always assume that $G$ has the following Taylor expansion near $u=0$ :

$$
G(u, \lambda)=G_{1}(u, \lambda)+o\left(\|u\|_{1}^{k}\right)
$$

for some integer $k \geq 2$, where $G_{1}: H_{1} \times \cdots \times H_{1} \rightarrow H$ is a $k$-multilinear mapping given by

$$
G_{1}(u, \lambda)=G_{1}(u, \cdots, u, \lambda)
$$

Let the eigenvalues (counting multiplicity) of $L_{\lambda}$ be real and be given by $\left\{\beta_{1}(\lambda), \beta_{2}(\lambda), \cdots\right\}$ with $\beta_{k}(\lambda) \in \mathbb{R}$ such that

$$
\begin{aligned}
& \beta_{i}(\lambda) \begin{cases}<0, & \lambda<\lambda_{0}, \\
=0, & \lambda=\lambda_{0}, \quad \forall 1 \leq i \leq m, \\
>0, & \lambda>\lambda_{0},\end{cases} \\
& \beta_{j}\left(\lambda_{0}\right)<0, \quad \forall m+1 \leq j .
\end{aligned}
$$

Let $\left\{e_{1}, \cdots, e_{r}\right\}$ and $\left\{e_{1}^{*}, \cdots, e_{r}^{*}\right\} \subset H_{1}$ be the eigenvectors of $L_{\lambda}$ and $L_{\lambda}^{*}$ at $\lambda=\lambda_{0}$ respectively:

$$
L_{\lambda_{0}} e_{j}=0, \quad L_{\lambda_{0}}^{*} e_{j}^{*}=0, \quad 1 \leq j \leq r .
$$

Here $r \leq m$ is the geometric multiplicity of the eigenvalue $\beta_{1}\left(\lambda_{0}\right)$.

We let

$$
a_{j_{1} \cdots j_{k}}^{i}=<G_{1}\left(e_{j_{1}}, \cdots, e_{j_{k}}, \lambda\right), e_{i}^{*}>_{H}
$$

Definition 3.1. Assume (3.2)-(3.5). The steady state solution $u=0$ of (3.1) is called $k$-th order nondegenerate at $\lambda=\lambda_{0}$, if $x=\left(x_{1}, x_{2}, \cdots, x_{r}\right)=0$ is an isolated singular point of the following system of $r$-dimensional algebraic equations

$$
\sum_{j_{1}, \cdots, j_{k}=1}^{r} a_{j_{1} \cdots j_{k}}^{i} x_{j_{1}} \cdots x_{j_{k}}=0, \quad 1 \leq i \leq r \quad \text { and } \lambda=\lambda_{0} .
$$

The following two theorems prove bifurcation from higher-order nondegenerate singular points. 
Theorem 3.2. Let the operator $L_{\lambda}+G: H_{1} \rightarrow H$ satisfy (3.2)-(3.5). If there is an even number $k \geq 2$ such that $u=0$ is a $k$-th order nondegenerate singular point of (3.1) at $\lambda=\lambda_{0}$, then $(u, \lambda)=\left(0, \lambda_{0}\right)$ must be a bifurcation point of (3.1), and there is at least one bifurcated branch on each side of $\lambda=\lambda_{0}$.

In this theorem, the condition that $k$ is even is needed. For example, the following system

$$
\left\{\begin{array}{l}
x_{1}-x_{2}^{3}=0 \\
x_{2}+x_{1}^{3}=0
\end{array}\right.
$$

which corresponds to the case where $m=2, r=2$, and $k=3$ odd, has no bifurcation.

This theorem establishes the existence of transcritical bifurcation, and the following is its global version, in the same spirit of the Rabinowitz global theorem. Let

$$
\begin{aligned}
& \Sigma^{+}=\left\{(u, \lambda) \in H_{1} \times \mathbb{R} \mid L_{\lambda} u+G(u, \lambda)=0, u \neq 0, \lambda>\lambda_{0}\right\}, \\
& \Sigma^{-}=\left\{(u, \lambda) \in H_{1} \times \mathbb{R} \mid L_{\lambda} u+G(u, \lambda)=0, u \neq 0, \lambda<\lambda_{0}\right\} .
\end{aligned}
$$

Theorem 3.3. Let $L: H_{1} \rightarrow H$ satisfy (2.32) and (2.33) $G(\cdot, \lambda): H_{1} \rightarrow H$ be compact, and assume the conditions of Theorem 3.2 hold true. Let $\Sigma \subset \bar{\Sigma}^{+}$(resp. $\left.\Sigma \subset \bar{\Sigma}^{-}\right)$be the connected component of $\bar{\Sigma}^{+}$(resp. $\left.\bar{\Sigma}^{-}\right)$containing $\left(0, \lambda_{0}\right)$. Then one of the following assertions hold true.

(1) $\Sigma$ is unbounded;

(2) $\Sigma$ contains points $\left(0, \lambda_{1}\right)$ with $\lambda_{1}>\lambda_{0}$ (resp. $\left.\lambda_{1}<\lambda_{0}\right)$ such that there are some eigenvalues $\beta_{j}(\lambda)$ of $L_{\lambda}$ at $\lambda=\lambda_{1}$, i.e. $\beta_{j}\left(\lambda_{1}\right)=0$;

(3) there exists a point $\left(v_{0}, \mu\right) \in H_{1} \times \mathbb{R}$ with $\mu>\lambda_{0}$ (resp. $\mu<\lambda_{0}$ ) such that

$$
\Sigma \cap H_{1} \times\{\lambda\}= \begin{cases}\emptyset, & \lambda>\mu \quad(\text { resp. } \lambda<\mu), \\ \left(v_{0}, \mu\right), & \lambda=\mu, \\ \Gamma_{1}(\lambda)+\Gamma_{2}(\lambda), & \lambda_{0} \leq \lambda<\mu \quad\left(\text { resp. } \lambda_{0} \geq \lambda>\mu\right),\end{cases}
$$

where $\Gamma_{1}\left(\lambda_{0}\right)=\left(0, \lambda_{0}\right),\left(0, \lambda_{0}\right) \notin \Gamma_{2}\left(\lambda_{0}\right), \Gamma_{1}(\lambda) \neq \emptyset$, and $\Gamma_{2}(\lambda) \neq \emptyset$.

3.2. Proof of Theorem 3.2. We proceed with the Lyapunov-Schmidt method, together with the Brouwer degree theory.

SteP 1. Consider (3.1). Let $\left\{w_{1}(\lambda), \cdots, w_{m}(\lambda)\right\} \quad \subset \quad H_{1}$ and $\left\{w_{1}^{*}(\lambda), \cdots, w_{m}^{*}(\lambda)\right\} \subset H_{1}$ be the eigenvectors of $L_{\lambda}$ and $L_{\lambda}^{*}$ respectively, corresponding to eigenvelues given by (3.4), i.e.

$$
\left(L_{\lambda}-\beta_{i}(\lambda)\right)^{n_{i}} w_{i}(\lambda)=0, \quad\left(L_{\lambda}^{*}-\beta_{i}(\lambda)\right)^{m_{i}} w_{i}^{*}(\lambda)=0,
$$

for some $n_{i}, m_{i} \geq 1(1 \leq i \leq m)$.

By Theorem 2.3, near $\lambda=\lambda_{0}$, the spaces $H_{1}$ and $H$ can be decomposed into the following direct sums

$$
\begin{aligned}
& H_{1}=E_{1}^{\lambda} \oplus E_{2}^{\lambda}, \quad \operatorname{dim} E_{1}^{\lambda}<\infty, \\
& H=\widetilde{E}_{1}^{\lambda} \oplus \widetilde{E}_{2}^{\lambda}, \\
& E_{1}^{\lambda}=\operatorname{span}\left\{w_{1}(\lambda), \cdots, w_{m}(\lambda)\right\}, \\
& E_{2}^{\lambda}=\left\{u \in H_{1} \mid<u, w_{i}^{*}(\lambda)>=0, i=1, \cdots, m\right\}, \\
& \widetilde{E}_{2}^{\lambda}=\text { closure of } E_{2}^{\lambda} \text { in } H,
\end{aligned}
$$


and $L_{\lambda}$ can be decomposed into $L_{\lambda}=\mathcal{L}_{1}^{\lambda} \oplus \mathcal{L}_{2}^{\lambda}$ such that for any $\lambda$ near $\lambda_{0}$,

$$
\begin{aligned}
& \mathcal{L}_{1}^{\lambda}=\left.L_{\lambda}\right|_{E_{1}^{\lambda}}: E_{1}^{\lambda} \rightarrow E_{1}^{\lambda}, \\
& \mathcal{L}_{2}^{\lambda}=\left.L_{\lambda}\right|_{E_{2}^{\lambda}}: E_{2}^{\lambda} \rightarrow \widetilde{E}_{2}^{\lambda} .
\end{aligned}
$$

Thus, near $\lambda_{0},(3.1)$ can be equivalently written as

$$
\begin{aligned}
& \mathcal{L}_{1}^{\lambda} v_{1}+P_{1} G\left(v_{1}+v_{2}, \lambda\right)=0, \\
& \mathcal{L}_{2}^{\lambda} v_{2}+P_{2} G\left(v_{1}+v_{2}, \lambda\right)=0,
\end{aligned}
$$

where $P_{1}: H \rightarrow E_{1}^{\lambda}$ and $P_{2}: H \rightarrow \widetilde{E}_{2}^{\lambda}$ are the canonical projections, $u=v_{1}+v_{2}$, $v_{1} \in E_{1}^{\lambda}$, and $v_{2} \in \widetilde{E}_{2}^{\lambda}$.

By (3.5), the mapping $\mathcal{L}_{2}^{\lambda}: E_{2}^{\lambda} \rightarrow \widetilde{E}_{2}^{\lambda}$ is a linear homeomorphism near $\lambda_{0}$. By the implicit function theorem, there exists a solution $v_{2}=f\left(v_{1}, \lambda\right)$ for (3.9). Then the bifurcation equation (3.1) is reduced to the following equation

$$
\mathcal{L}_{1}^{\lambda} v+P_{1} G(v+f(v, \lambda), \lambda)=0, \quad v \in E_{1}^{\lambda} .
$$

It follows from (3.2) and (3.9) that

$$
f(v, \lambda)=o(\|v\|), \quad \forall \lambda \in \mathbb{R} .
$$

Therefore, by (3.2) and (3.11), the nonlinear operator $g_{\lambda}=P_{1} G: E_{1}^{\lambda} \rightarrow E_{1}^{\lambda}$ can be expressed as

$$
g_{\lambda}=P_{1} G(v+f(v, \lambda), \lambda)=P_{1} G_{1}(v, \lambda)+\widetilde{g}(v, \lambda),
$$

where

$$
\widetilde{g}(v, \lambda)=o\left(\|v\|^{k}\right) .
$$

Since the eigenvalues of $\mathcal{L}_{1}^{\lambda}$ are given by $\beta_{i}(\lambda)(1 \leq i \leq m)$ satisfying (3.4), we have the following index formula

$$
\operatorname{ind}\left(\mathcal{L}_{1}^{\lambda}+g_{\lambda}, 0\right)= \begin{cases}(-1)^{m}, & \lambda<\lambda_{0} \\ 1, & \lambda>\lambda_{0} .\end{cases}
$$

To complete the proof of the theorem, it suffices to show that

$$
\operatorname{ind}\left(\mathcal{L}_{1}^{\lambda_{0}}+g_{\lambda_{0}}, 0\right)=\text { even }
$$

We proceed in three steps to prove (3.14).

STEP 2. We first consider the case where $\mathcal{L}_{1}^{\lambda_{0}}=0$, i.e. $r=m$, the geometric multiplicity equals to the algebraic multiplicity at $\lambda_{0}$.

By assumptions, $P_{1} G_{1}: E_{1}^{\lambda_{0}} \rightarrow E_{1}^{\lambda_{0}}$ is a nondegenerate k-th order multilinear mapping. There exists a number $\beta>0$ such that

$$
\left\|P_{1} G_{1}\left(\alpha v, \lambda_{0}\right)\right\| \geq \beta|\alpha|^{k}, \quad \forall v \in E_{1}^{\lambda_{0}},\|v\|=1 .
$$

It follows from (3.12) and (3.15) that for any $R>0$ sufficiently small,

$$
P_{1} G_{1}\left(v, \lambda_{0}\right)+t \widetilde{g}\left(v, \lambda_{0}\right) \neq 0, \forall v \in E_{1}^{\lambda_{0}},\|v\|=R, \quad 0 \leq t \leq 1 .
$$


By the homotopy invariance property of the Brouwer degree, we have

$$
\begin{aligned}
\operatorname{ind}\left(\mathcal{L}_{1}^{\lambda_{0}}+g_{\lambda_{0}}, 0\right) & =\operatorname{deg}\left(\mathcal{L}_{1}^{\lambda_{0}}+g_{\lambda_{0}}, B_{R}, 0\right) \\
& =\operatorname{deg}\left(P_{1} G_{1}\left(\cdot, \lambda_{0}\right), B_{R}, 0\right),
\end{aligned}
$$

where $B_{R}=\left\{v \in E_{1}^{\lambda_{0}} \mid\|v\|<R\right\}$.

When $k$ is even in (3.3), the degree in (3.16) must be even. In fact, by the Sard theorem, there exists a $w \in E_{1}^{\lambda_{0}}$ with $\|w\| \neq 0$ sufficiently small such that $w$ is a regular value of $P_{1} G_{1}$. Thus, the equation

$$
P_{1} G_{1}\left(v, \lambda_{0}\right)=w
$$

has either no solution, or even number of solutions

$$
\pm v_{1}, \cdots, \pm v_{n} \in B_{R} .
$$

Notice that the Jacobian of $P_{1} G_{1}$ is odd, i.e.

$$
J P_{1} G_{1}(-v, \lambda)=-J P_{1} G_{1}(v, \lambda), \quad \forall v \in E_{1}^{\lambda_{0}} .
$$

Then we have

$$
\begin{aligned}
\operatorname{deg} & \left.P_{1} G_{1}\left(\cdot, \lambda_{0}\right), B_{R}, 0\right) \\
& =\sum_{j=1}^{n} \operatorname{sign} \operatorname{det} J P_{1} G_{1}\left( \pm v_{j}, \lambda_{0}\right) \\
& = \begin{cases}0, & \text { as } m=\text { odd }, \\
2 \sum_{j=1}^{n} \operatorname{sign} \operatorname{det} J P_{1} G_{1}\left(v_{j}, \lambda_{0}\right), & \text { as } m=\text { even. }\end{cases}
\end{aligned}
$$

Hence, (3.14) follows from (3.16) and (3.17).

Step 3. The Case where $m=3, r=2$ and $k=2$. To present the main idea of the proof, we proceed with the special case where the algebraic multiplicity $m=3$ and the geometric multiplicity $r=2$ at $\lambda=\lambda_{0}$.

Let

$$
\begin{aligned}
& E_{1}^{\lambda_{0}}=\operatorname{span}\left\{w_{1}, w_{2}, w_{3}\right\} \\
& \mathcal{L}_{1}^{\lambda_{0}} w_{1}=0 \\
& \mathcal{L}_{1}^{\lambda_{0}} w_{2}=0 \\
& \mathcal{L}_{1}^{\lambda_{0}} w_{3}=w_{2} .
\end{aligned}
$$

By (2.22) and (2.23) in the proof of Theorem 2.3, we see that the dual eigenvectors of $\left\{w_{1}, w_{2}, w_{3}\right\}$ satisfying $<w_{i}, w_{j}^{*}>=\delta_{i j}$ are given by

$$
\begin{aligned}
& \mathcal{L}_{1}^{*} \lambda_{0} w_{1}^{*}=0, \\
& \mathcal{L}_{1}^{*} \lambda_{0} w_{2}^{*}=w_{3}^{*}, \\
& \mathcal{L}_{1}^{*} \lambda_{0} w_{3}^{*}=0 .
\end{aligned}
$$


Therefore, we have the following

$$
e_{1}=w_{1}, \quad e_{2}=w_{2}, \quad e_{1}^{*}=w_{1}^{*}, \quad e_{2}^{*}=w_{3}^{*} .
$$

Under the basis $\left\{w_{1}, w_{2}, w_{3}\right\}$, we have

$$
\mathcal{L}_{1}^{\lambda_{0}} v=\left(\begin{array}{ccc}
0 & 0 & 0 \\
0 & 0 & 1 \\
0 & 0 & 0
\end{array}\right)\left(\begin{array}{l}
x_{1} \\
x_{2} \\
x_{3}
\end{array}\right), \quad v=\sum_{i=1}^{3} x_{i} w_{i}=\left(\begin{array}{l}
x_{1} \\
x_{2} \\
x_{3}
\end{array}\right)
$$

and, by $k=2$,

$$
P_{1} G_{1}(v)=\left(\begin{array}{c}
\sum_{i, j=1}^{3} b_{i j}^{1} x_{i} x_{j} \\
\sum_{i, j=1}^{3} b_{i j}^{2} x_{i} x_{j} \\
\sum_{i, j=1}^{3} b_{i j}^{3} x_{i} x_{j}
\end{array}\right), \quad b_{i j}^{l}=<G_{1}\left(w_{i}, w_{j}\right), w_{l}^{*}>_{H} .
$$

Let $T: E_{1}^{\lambda_{0}} \rightarrow E_{1}^{\lambda_{0}}$ be a linear homeomorphism defined by

$$
\begin{aligned}
& T v=\left(\begin{array}{lll}
1 & 0 & 0 \\
0 & 0 & 1 \\
0 & 1 & 0
\end{array}\right)\left(\begin{array}{c}
x_{1} \\
x_{2} \\
x_{3}
\end{array}\right), \\
& T \mathcal{L}_{1}^{\lambda_{0}} v=x_{3} w_{3}=\left(\begin{array}{c}
0 \\
0 \\
x_{3}
\end{array}\right) .
\end{aligned}
$$

Then we derive from (3.19), (3.20) and (3.12) that

$$
\begin{aligned}
& T\left(\mathcal{L}_{1}^{\lambda_{0}}+g_{\lambda_{0}}\right)(v)=T\left(\mathcal{L}_{1}^{\lambda_{0}}+P_{1} G_{1}\right)(v)+T \widetilde{g}\left(v, \lambda_{0}\right), \\
& T\left(\mathcal{L}_{1}^{\lambda_{0}}+P_{1} G_{1}\right)(v)=\left(\begin{array}{c}
\sum_{i, j=1}^{3} b_{i j}^{1} x_{i} x_{j} \\
\sum_{i, j=1}^{3} b_{i j}^{3} x_{i} x_{j} \\
x_{3}+\sum_{i, j=1}^{3} b_{i j}^{2} x_{i} x_{j}
\end{array}\right) \\
& T \widetilde{g}\left(v, \lambda_{0}\right)=o\left(\sum_{i=1}^{3} x_{i}^{2}\right) .
\end{aligned}
$$

By assumption, $v_{0}=x_{1} e_{1}+x_{2} e_{2}=0$ is an isolated singular point of the following equations

$$
\sum_{i, j=1}^{2} a_{i j}^{l} x_{i} x_{j}=0, \quad l=1,2
$$


where

$$
\begin{aligned}
& a_{i j}^{1}=<G_{1}\left(e_{i}, e_{j}\right), e_{1}^{*}>_{H}=b_{i j}^{1}, \\
& a_{i j}^{2}=<G_{1}\left(e_{i}, e_{j}\right), e_{2}^{*}>_{H}=b_{i j}^{3} .
\end{aligned}
$$

Thus we infer from (3.22) and (3.23) that for any $0 \leq t \leq 1, v=\sum_{i=1}^{3} x_{i} w_{i}=0$ is an isolated zero point of the equation

$$
\left(\begin{array}{c}
\sum_{i, j=1}^{2} a_{i j}^{1} x_{i} x_{j}+t x_{3} \sum_{i=1}^{2}\left(b_{i 3}^{1}+b_{3 i}^{1}\right) x_{i}+t b_{33}^{1} x_{3}^{2} \\
\sum_{i, j=1}^{2} a_{i j}^{2} x_{i} x_{j}+t x_{3} \sum_{i=1}^{2}\left(b_{i 3}^{3}+b_{3 i}^{3}\right) x_{i}+t b_{33}^{3} x_{3}^{2} \\
x_{3}+t \sum_{i, j=1}^{3} b_{i j}^{2} x_{i} x_{j}
\end{array}\right)+t T \widetilde{g}\left(v, \lambda_{0}\right)=0 .
$$

Based on the homotopy invariance property of the Brouwer degree theory, it follows from (3.21)-(3.24) that

$$
\operatorname{deg}\left(T\left(\mathcal{L}_{1}^{\lambda_{0}}+g_{\lambda_{0}}\right), B_{R}, 0\right)=\operatorname{deg}\left(K, B_{R}, 0\right),
$$

where $R>0$ is sufficiently small, and

$$
K(v)=\left(\begin{array}{c}
\sum_{i, j=1}^{2} a_{i j}^{1} x_{i} x_{j} \\
\sum_{i, j=1}^{2} a_{i j}^{2} x_{i} x_{j} \\
x_{3}
\end{array}\right) .
$$

According to the multiplication theorem and the dimension reduction theorem of the Brouwer degree theory, it follows from (3.21)-(3.24) that

$$
\begin{aligned}
\operatorname{deg}\left(T\left(\mathcal{L}_{1}^{\lambda_{0}}+g_{\lambda_{0}}\right), B_{R}, 0\right) & =\operatorname{ind}(T, 0) \cdot \operatorname{deg}\left(\mathcal{L}_{1}^{\lambda_{0}}+g_{\lambda_{0}}, B_{R}, 0\right) \\
& =(-1) \operatorname{deg}\left(\mathcal{L}_{1}^{\lambda_{0}}+g_{\lambda_{0}}, B_{R}, 0\right),
\end{aligned}
$$

and

$$
\operatorname{deg}\left(K, B_{R}, 0\right)=\operatorname{deg}\left(\widetilde{K}, B_{R} \cap E, 0\right),
$$

where $E=\operatorname{span}\left\{e_{1}, e_{2}\right\}$, and

$$
\widetilde{K}\left(v_{0}\right)=\left(\begin{array}{c}
\sum_{i, j=1}^{2} a_{i j}^{1} x_{i} x_{j} \\
\sum_{i, j=1}^{2} a_{i j}^{2} x_{i} x_{j}
\end{array}\right), \quad v_{0}=x_{1} e_{1}+x_{2} e_{2}=\left(x_{1}, x_{2}\right)^{t} .
$$

By assumption, $v_{0}=0$ is an isolated singular point of $\widetilde{K}$. Hence we can prove in the same fashion as in Step 2 that

$$
\operatorname{deg}\left(\widetilde{K}, B_{R} \cap E, 0\right)=\text { even. }
$$


Finally, the index formula (3.24) follows from (3.25)-(3.28).

SteP 4. The General CASE. Let the operator $\mathcal{L}_{1}^{\lambda_{0}}: E_{1}^{\lambda_{0}} \rightarrow E_{1}^{\lambda_{0}}$ have the following Jordan form

$$
\mathcal{L}_{1}^{\lambda_{0}}=\left(\begin{array}{cccc}
0 & 0 & \cdots & 0 \\
0 & J_{1} & \cdots & 0 \\
\vdots & \vdots & & \vdots \\
0 & 0 & \cdots & J_{n}
\end{array}\right), \quad J_{i}=\left(\begin{array}{ccccc}
0 & 1 & 0 & \cdots & 0 \\
0 & 0 & 1 & \cdots & 0 \\
\vdots & \vdots & \vdots & & \vdots \\
0 & 0 & 0 & \cdots & 1 \\
0 & 0 & 0 & \cdots & 0
\end{array}\right)
$$

The space $E_{1}^{\lambda_{0}}$ can be decomposed into

$$
E_{1}^{\lambda_{0}}=E \oplus V, \quad E=\operatorname{span}\left\{e_{1}, \cdots, e_{r}\right\},
$$

and $V \subset E_{1}^{\lambda_{0}}$ is the complement of $E$.

It is known that there exists a linear homeomorphism $T: E_{1}^{\lambda_{0}} \rightarrow E_{1}^{\lambda_{0}}$ such that

$$
\begin{aligned}
& \left.T \mathcal{L}_{1}^{\lambda_{0}}\right|_{E}=0,\left.\quad T \mathcal{L}_{1}^{\lambda_{0}}\right|_{V}=I_{m-r} \text { the identify matrix, } \\
& \left.P T P_{1} G_{1}\right|_{E}: E \rightarrow E \\
& <G_{1}\left(e_{j_{1}}, \cdots, e_{j_{k}}, \lambda_{0}\right), e_{i}^{*}>_{H} \\
& \quad=<P T P_{1} G_{1}\left(e_{j_{1}}, \cdots, e_{j_{k}}, \lambda_{0}\right), w_{i}^{*}>_{H}
\end{aligned}
$$

for $1 \leq i \leq r$, where $P: E_{1}^{\lambda_{0}} \rightarrow E$ is the projection, $\left\{w_{1}^{*}, \cdots, w_{r}^{*}\right\}$ are the dual eigenvectors of $\left\{e_{1}, \cdots, e_{r}\right\}$, i.e.

$$
<e_{i}, w_{j}^{*}>_{H}=\delta_{i j} .
$$

As in the proof of (3.25)-(3.28), we infer from (3.29)-(3.31) that

$$
\begin{aligned}
\operatorname{deg}\left(\mathcal{L}_{1}^{\lambda_{0}}+g_{\lambda_{0}}, B_{R}, 0\right) & =(-1)^{m-r} \operatorname{deg}\left(T\left(\mathcal{L}_{1}^{\lambda_{0}}+g_{\lambda_{0}}\right), B_{R}, 0\right) \\
& =(-1)^{m-r} \operatorname{deg}\left(T \mathcal{L}_{1}^{\lambda_{0}}+T P_{1} G_{1}, B_{R}, 0\right) \\
& =(-1)^{m-r} \operatorname{deg}\left(P T P_{1} G_{1}, B_{R} \cap E, 0\right) .
\end{aligned}
$$

As for (3.17), we can show that

$$
\operatorname{deg}\left(P T P_{1} G_{1}, B_{R} \cap E, 0\right)=\text { even, }
$$

and (3.14) follows.

The proof of the theorem is complete.

3.3. Proof of Theorem 3.3. It is easy to see that the Leray-Schauder degree theory is valid for the completely continuous fields $L_{\lambda}+G: H_{1} \rightarrow H$, provided that $L$ satisfies (2.32) and (2.33). We know that the eigenvalues $\beta_{j}(\lambda)$ of $L_{\lambda}$ with $\operatorname{Re} \beta_{j}(\lambda) \geq 0$ are finite, and if $L_{\lambda}: H_{1} \rightarrow H$ is invertible, then

$$
\operatorname{ind}\left(-\left(L_{\lambda}+G\right), 0\right)=\operatorname{deg}\left(-\left(L_{\lambda}+G\right), B_{r}, 0\right)=(-1)^{n},
$$

where $n$ is the sum of all algebraic multiplicities of the real eigenvalues $\beta_{j}(\lambda)>0$ of $L_{\lambda}, B_{r}=\left\{u \in H_{1} \mid\|u\|_{H_{1}}<r\right\}$, and $r>0$ sufficiently small. 
By similar properties for the Leray-Schauder degree theory as those for the Brouwer degree used in the proof of Theorem 3.2, we can show that under the conditions of Theorem 3.3, the topological degree of $L_{\lambda}+G$ at $\lambda=\lambda_{0}$ is an even number, i.e.

$$
\begin{aligned}
\operatorname{deg}\left(-\left(L_{\lambda}+G\right), B_{r}, 0\right) & =(-1)^{m} \operatorname{deg}\left(T P_{1} G_{1}, B_{r} \cap E, 0\right) \\
& =\text { even, }
\end{aligned}
$$

where $E, T P_{1} G_{1}$ are the same as in (3.33), and $n \geq 0$ is defined as in (3.34).

Then the rest of the proof of the theorem is routine using (3.34) and (3.35). The proof is complete.

REMARK 3.4. In fact, in the proof of Theorem 3.2 and 3.3, we have shown the following result for the Leray-Schauder degree.

Let $L_{\lambda}+G: H_{1} \rightarrow H$ be a completely continuous field, and $L_{\lambda}$ satisfy (2.32) and (2.33). If there are $\left\{w_{1}, \cdots, w_{m}\right\} \subset H_{1}$ such that

$$
L_{\lambda_{0}}^{n_{i}} w_{i}=0, \quad 1 \leq i \leq m, \quad n_{i} \geq 1,
$$

and $u=0$ is a k-th order nondegenerate singular point of $L_{\lambda_{0}}+G\left(\cdot, \lambda_{0}\right)$ for some $k \geq 2$, then

$$
\operatorname{deg}\left(-\left(L_{\lambda_{0}}+G\left(\cdot, \lambda_{0}\right)\right), B_{r}, 0\right)=(-1)^{n} \operatorname{deg}\left(\mathcal{L}^{\lambda_{0}}+g, B_{r} \cap E, 0\right),
$$

where $n$ is the sum of all algebraic multiplicities of the real eigenvalues $\beta_{j}\left(\lambda_{0}\right)>0$ of $L_{\lambda_{0}}, E=\operatorname{span}\left\{w_{1}, \cdots, w_{m}\right\}, \mathcal{L}^{\lambda_{0}}=-\left.L_{\lambda_{0}}\right|_{E}: E \rightarrow E, g=-\left.P G\right|_{E}: E \rightarrow E$, and $P: H \rightarrow E$ the canonical projection.

4. Bifurcation at geometric simple eigenvalues: $r=1$. In this section, we address the case where the geometric multiplicity of the eigenvalue is one, i.e. $r=1$ in (3.6).

Definition 4.1. Let $\Gamma(\lambda) \subset H_{1}$ be a branch bifurcated from $\left(u_{0}, \lambda_{0}\right)$ of (3.1). $\Gamma(\lambda)$ is called regular if for any $\left|\lambda-\lambda_{0}\right|>0$ sufficiently small each singular point $v_{\lambda} \in \Gamma(\lambda)$ of (3.1) is nondegenerate.

The main result in this section is as follows.

Theorem 4.2. Assume the conditions (3.2)- (3.5) and $r=1$ in (3.6). If the number

$$
\alpha=<G_{1}\left(e_{1}, \lambda_{0}\right), e_{1}^{*}>_{H} \neq 0,
$$

where $G_{1}$ is given by (3.3), then the following assertions hold true.

(1) For the case where $k$ is even in (3.3), there exists a unique bifurcated branch of (3.1) on each side of $\lambda_{0}$.

(2) For the case where $k$ is odd and $m$ is even, if $\alpha<0$, then (3.1) has no bifurcation from $\left(0, \lambda_{0}\right)$, and if $\alpha>0$, then there are exactly two branches bifurcated from $\left(0, \lambda_{0}\right)$ on each side of $\lambda_{0}$.

(3) For the case where $k$ is odd and $m$ is odd, (3.1) has no bifurcated branch on $\lambda<\lambda_{0}$ (resp. on $\lambda>\lambda_{0}$ ) and has exactly two branches on $\lambda>\lambda_{0}$ (resp. on $\left.\lambda<\lambda_{0}\right)$ if $\alpha<0$ (resp. $\left.\alpha>0\right)$.

(4) Each branch $\Gamma(\lambda)$ bifurcated from $\left(0, \lambda_{0}\right)$ for (3.1) is regular, and the singular points $u_{\lambda} \in \Gamma(\lambda)$ can be expressed as 


$$
\begin{aligned}
u_{\lambda}= \pm & \left|\alpha^{-1} \beta_{1}(\lambda) \cdots \beta_{m}(\lambda)\right|^{1 /(k-1)} e_{1} \\
& +o\left(\left|\alpha^{-1} \beta_{1}(\lambda) \cdots \beta_{m}(\lambda)\right|^{1 /(k-1)}\right) .
\end{aligned}
$$

Proof. Let $\left\{w_{1}, \cdots, w_{m}\right\}$ and $\left\{w_{1}^{*}, \cdots, w_{m}^{*}\right\} \subset H_{1}$ satisfy

$$
\left\{\begin{array}{l}
L_{\lambda_{0}} w_{1}=0, \quad L_{\lambda_{0}} w_{2}=w_{1}, \cdots, L_{\lambda_{0}} w_{m}=w_{m-1}, \\
L_{\lambda_{0}}^{*} w_{m}^{*}=0, \quad L_{\lambda_{0}}^{*} w_{m-1}^{*}=w_{m}^{*}, \cdots, L_{\lambda_{0}} w_{1}^{*}=w_{2}^{*} .
\end{array}\right.
$$

From the proof of Theorem 2.3, we see that

$$
<w_{i}, w_{j}^{*}>_{H}=\delta_{i j} .
$$

Applying the Lyapunov-Schmidt method as in the proof of Theorem 3.2, the bifurcation of (3.1) is equivalent to the following equation

$$
\begin{gathered}
\left(\begin{array}{ccccc}
\beta_{1}(\lambda) & 1 & 0 & \cdots & 0 \\
0 & \beta_{2}(\lambda) & 1 & \cdots & 0 \\
\vdots & \vdots & \vdots & & \vdots \\
0 & 0 & 0 & \cdots & \beta_{m}(\lambda)
\end{array}\right)\left(\begin{array}{c}
x_{1} \\
\vdots \\
x_{m}
\end{array}\right) \\
+P_{1} G(v+f(v, \lambda), \lambda)=0
\end{gathered}
$$

where $v=\sum_{i=1}^{m} x_{i} w_{i}(\lambda)$, and $f(v, \lambda)$ is given by (3.11).

We see from (4.3) that $e_{1}=w_{1}\left(\lambda_{0}\right)$, and $e_{1}^{*}=w_{m}^{*}\left(\lambda_{0}\right)$. By (3.1), (3.2) and (4.4), we infer from (4.5) that

$$
\left\{\begin{array}{l}
x_{2}=-\beta_{1}(\lambda) x_{1}+O\left(|x|^{k}\right), \\
\vdots \\
x_{m}=-\beta_{m-1}(\lambda) x_{m-1}+O\left(|x|^{k}\right), \\
\alpha(\lambda) x_{1}^{k}=-\beta_{m}(\lambda) x_{m}+O\left(\sum_{i=0}^{k-1} \sum_{j=2}^{m}\left|x_{1}\right|^{i}\left|x_{j}\right|^{k-i}\right),
\end{array}\right.
$$

where $\alpha\left(\lambda_{0}\right)=\alpha$. It follows from (4.6) that near

$$
\alpha(\lambda) x_{1}^{k-1}=(-1)^{m} \beta_{1}(\lambda) \cdots \beta_{m}(\lambda)+o\left(\left|\beta_{1}(\lambda) \cdots \beta_{m}(\lambda)\right|\right) .
$$

Thus, Assertions (1)-(3) and (4.2) follows from (3.4), (4.1) and (4.7).

Assertion (4) can be derived from the following determinant of the first order approximation of the Jacobian of (4.5):

$$
\begin{aligned}
& \operatorname{det}\left(\begin{array}{ccccc}
\beta_{1}(\lambda) & 1 & 0 & \cdots & 0 \\
0 & \beta_{2}(\lambda) & 1 & \cdots & 0 \\
\vdots & \vdots & \vdots & & \vdots \\
\alpha k x_{1}^{k-1} & 0 & 0 & \cdots & \beta_{m}(\lambda)
\end{array}\right) \\
& =\beta_{1} \cdots \beta_{m}-(-1)^{m} k \alpha x_{1}^{k-1} \\
& =\beta_{1} \cdots \beta_{m}+o\left(\left|\beta_{1}(\lambda) \cdots \beta_{m}(\lambda)\right|\right)(\text { by }(4.7)) \\
& \neq 0 \quad \text { for } \lambda \neq \lambda_{0}(\text { by }(3.4)) .
\end{aligned}
$$

The proof of the theorem is complete. $\square$ 
5. Bifurcation with $r=k=2$. Let $r=2$ in (3.6), $G_{1}$ is bilinear (i.e. $k=2$ ), and

$$
\begin{aligned}
& a_{11}=<G_{1}\left(e_{1}, e_{1}, \lambda\right), e_{1}^{*}>_{H}, \\
& a_{22}=<G_{1}\left(e_{2}, e_{2}, \lambda\right), e_{1}^{*}>_{H}, \\
& a_{12}=<G_{1}\left(e_{1}, e_{2}, \lambda\right), e_{1}^{*}>_{H}+<G_{1}\left(e_{2}, e_{1}, \lambda\right), e_{1}^{*}>_{H}, \\
& b_{11}=<G_{1}\left(e_{1}, e_{1}, \lambda\right), e_{2}^{*}>_{H}, \\
& b_{22}=<G_{1}\left(e_{2}, e_{2}, \lambda\right), e_{2}^{*}>_{H}, \\
& b_{12}=<G_{1}\left(e_{1}, e_{2}, \lambda\right), e_{2}^{*}>_{H}+<G_{1}\left(e_{2}, e_{1}, \lambda\right), e_{2}^{*}>_{H} .
\end{aligned}
$$

We have the following bifurcation theorem.

TheOREm 5.1. Let the conditions (3.1)-(3.5) and $r=2$ in (3.6) hold true. If $u=0$ is a second-order nondegenerate singular point of $L_{\lambda}+G$ at $\lambda=\lambda_{0}$, and the two vectors $\left(a_{11}, a_{12}, a_{22}\right)$ and $\left(b_{11}, b_{12}, b_{22}\right)$ are linearly independent, then we have the following assertions.

(1) There are at most three bifurcated branches of (3.1) bifurcated from $\left(0, \lambda_{0}\right)$ on each side of $\lambda=\lambda_{0}$.

(2) If the bifurcated branches on $\lambda>\lambda_{0}$ (resp. on $\lambda<\lambda_{0}$ ) are regular, then the number of branches on this side is either 1 or 3.

(3) On any given side, if the number of of branches is 3 , then these 3 branches must be regular.

Proof. We proceed in several steps as follows.

STEP 1 . We only have to prove the case where $m=r=2$. For the case where $m>r=2$, as in the proof of Theorem 4.2, the $m$-dimensional bifurcation equations (3.10) can be treated as if it is two-dimensional equations, and the proof given below for the case where $m=r=2$ can then be applied as well.

As $m=r=2$ in (3.4) and (3.6), the bifurcation equations (3.10) can be written as follows

$$
\left\{\begin{array}{l}
\beta_{1}(\lambda) x_{1}+a_{11} x_{1}^{2}+a_{12} x_{1} x_{2}+a_{22} x_{2}^{2}+o\left(|x|^{2}\right)=0 \\
\beta_{2}(\lambda) x_{2}+b_{11} x_{1}^{2}+b_{12} x_{1} x_{2}+b_{22} x_{2}^{2}+o\left(|x|^{2}\right)=0
\end{array}\right.
$$

where $x=\left(x_{1}, x_{2}\right)^{t}$.

STEP 2. We now show that there is an $\epsilon>0$ such that for any function $\left(f_{1}(x), f_{2}(x)\right)$ with $\left|f_{1}(x)\right|+\left|f_{2}(x)\right|$ small near $x=0$, the following equations

$$
\begin{aligned}
& a_{11} x_{1}^{2}+a_{12} x_{1} x_{2}+a_{22} x_{2}^{2}+f_{1}(x)+o\left(|x|^{2}\right)=0, \\
& b_{11} x_{1}^{2}+b_{12} x_{1} x_{2}+b_{22} x_{2}^{2}+f_{2}(x)+o\left(|x|^{2}\right)=0,
\end{aligned}
$$

have at most four solutions in $|x|<\epsilon$.

Since the second order terms in (5.2) and (5.3) are non-degenerate, at least one of the coefficients $a_{11}, a_{22}, b_{11}$, and $b_{22}$ is not zero. Without loss of generality, we assume that $a_{22} \neq 0$. Then we obtain from (5.2) that

$$
x_{2}^{ \pm}=-\frac{a_{12}}{2 a_{22}} x_{1} \pm \frac{1}{2 a_{22}} \sqrt{\left(a_{12}^{2}-4 a_{11} a_{22}\right) x_{1}^{2}-4 a_{22} f_{1}(x)+o\left(|x|^{2}\right)},
$$


which, by the implicit function theorem, implies that

$$
x_{2}^{ \pm}=-\frac{a_{12}}{2 a_{22}} x_{1} \pm \frac{1}{2 a_{22}} \triangle,
$$

where

$$
\begin{aligned}
& \triangle=\sqrt{\left(a_{12}^{2}-4 a_{11} a_{22}\right) x_{1}^{2}+g\left(x_{1}\right)+o\left(\left|x_{1}\right|^{2}\right)}, \\
& g\left(x_{1}\right) \rightarrow 0 \text { as } f_{1}(x) \rightarrow 0 .
\end{aligned}
$$

Inserting (5.5) into (5.3), we have

$$
\alpha x_{1}^{2}+b_{22} g\left(x_{1}\right)+4 a_{22}^{2} f_{2}(x)+o\left(|x|^{2}\right)=\mp \beta x_{1} \triangle,
$$

where

$$
\begin{aligned}
& \alpha=4 a_{22}\left(a_{22} b_{11}-a_{11} b_{22}\right)+2 a_{12}\left(a_{12} b_{22}-a_{22} b_{12}\right), \\
& \beta=2\left(a_{22} b_{12}-a_{12} b_{22}\right) .
\end{aligned}
$$

We infer then from (5.5) and (5.6) that

$$
\rho x_{1}^{4}+h_{1}\left(x_{1}\right) x_{1}^{2}+h_{2}\left(x_{1}\right)+o\left(|x|^{4}\right)=0,
$$

where

$$
\begin{aligned}
& \rho=\alpha^{2}-\beta^{2}\left(a_{11}^{2}-4 a_{11} a_{22}\right), \\
& h_{1}\left(x_{1}\right), h_{2}\left(x_{1}\right) \rightarrow 0 \text { as } f_{1}(x), f_{2}(x) \rightarrow 0 .
\end{aligned}
$$

It is easy to see that the condition $\rho \neq 0$ is equivalent to the following conditions

$$
b_{22} z_{ \pm}^{2}+b_{12} z_{ \pm}+b_{11} \neq 0, \quad z_{ \pm}=\frac{-a_{12} \pm \sqrt{a_{12}^{2}-4 a_{11} a_{22}}}{2 a_{22}} .
$$

By the assumptions of this theorem, conditions in (5.8) are valid. Therefore, $\rho \neq 0$, which implies that (5.7) has at most four real solutions satisfying that $x_{1} \rightarrow 0$ as $f_{1}(x), f_{2}(x) \rightarrow 0$.

On the other hand, each solution of (5.7) corresponds to one of the signs \pm in (5.5) and (5.6). Hence equations (5.2) and (5.3) have at most four real solutions near $x=0$. Namely, Assertion (1) is proved.

Step 3. Proof of Assertion (2). Let $F_{\lambda}: \mathbb{R}^{2} \rightarrow \mathbb{R}^{2}$ be defined by

$$
F_{\lambda}(x)=\left(\begin{array}{c}
\beta_{1}(\lambda) x_{1}+a_{11} x_{1}^{2}+a_{12} x_{1} x_{2}+a_{22} x_{2}^{2}+o\left(|x|^{2}\right) \\
\beta_{2}(\lambda) x_{2}+b_{11} x_{1}^{2}+b_{12} x_{1} x_{2}+b_{22} x_{2}^{2}+o\left(|x|^{2}\right)
\end{array}\right) .
$$

It is clear that the regularity of the bifurcated branches of (3.1) is equivalent to the regularity of singular points of (5.1). Hence for the singular points $z(\lambda)$ near $x=0$, we have

$$
\operatorname{ind}\left(F_{\lambda}, z(\lambda)\right)= \pm 1
$$

for $\left|\lambda-\lambda_{0}\right| \neq 0$ sufficiently small. In addition, we know that

$$
\operatorname{ind}\left(F_{\lambda}, 0\right)=\operatorname{sign} \beta_{1}(\lambda) \beta_{2}(\lambda)=1,
$$


as $\lambda \neq \lambda_{0}$, and by (3.14), we have

$$
\sum_{\left|z_{i}\right|<\epsilon} \operatorname{ind}\left(F_{\lambda}, z_{i}(\lambda)\right)=\text { even }
$$

for any $\epsilon>0$ small. Hence Assertion (2) follows from (5.9)-(5.11) and Assertion (1).

Step 4. Proof of Assertion (3). It suffices to show that the bifurcated singular points of $(5.1)$ are regular. Let $z_{i}(\lambda) \in \mathbb{R}^{2}(i=1,2,3)$ be the bifurcated singular points of (5.1).

Suppose that $z_{1}(\lambda)$ is degenerate, i.e.

$$
\operatorname{det} J F_{\lambda}\left(z_{1}(\lambda)\right)=0
$$

where $J F_{\lambda}$ stands for the Jacobian matrix of $F_{\lambda}$.

By (5.12), under suitable coordinate system, the function $F_{\lambda}$ can be expressed near $z_{1}(\lambda)$ as

$$
\widetilde{F}_{\lambda}(y)=\left(\begin{array}{c}
\alpha_{\lambda} y_{2}+c_{11} y_{1}^{2}+c_{12} y_{1} y_{2}+c_{22} y_{2}^{2}+o\left(|y|^{2}\right) \\
d_{11} y_{1}^{2}+d_{12} y_{1} y_{2}+d_{22} y_{2}^{2}+o\left(|y|^{2}\right)
\end{array}\right)
$$

where $y=x-z_{1}(\lambda)$. Hence the singular points of $\widetilde{F}_{\lambda}(y)$ near $y=0$ are given by

$$
y_{1}=0, \quad y_{2}=z_{2}-z_{1}, \quad y_{3}=z_{3}-z_{1}, \quad y_{4}=-z_{1} .
$$

Let $r>0$ be sufficiently small such that

$$
y_{2}, y_{3}, y_{4} \notin B_{2 r}=\left\{y \in \mathbb{R}^{2}|| y \mid<2 r\right\} .
$$

We take a $C^{\infty}$ cut-off function $\rho_{r}: \mathbb{R}^{2} \rightarrow \mathbb{R}$ as follows

$$
\rho_{r}(y)= \begin{cases}1, & \text { as } y \in B_{r}, \\ 0, & \text { as } y \in \mathbb{R}^{2} / B_{2 r} .\end{cases}
$$

By assumption, the quadratic form in $F_{\lambda}(x)$ is nondegenerate. Hence the quadratic form in $\widetilde{F}_{\lambda}(y)$ is also nondegenerate. Now we consider the perturbation equation

$$
\widetilde{F}_{\lambda}(y)+\rho_{r}(y)\left(\epsilon_{1} y_{1}, \epsilon_{2} y_{2}\right)^{t}=0
$$

for $\epsilon_{1}, \epsilon_{2}$ small.

It is clear that $y_{i}(1 \leq i \leq 4)$ are also solutions of (5.14). When $\alpha_{\lambda}=0$ in (5.13), by Theorem 3.2, for $\epsilon_{1}, \epsilon_{2}$ small, equation (5.14) has at least five solutions near $y=0$. When $\alpha_{\lambda} \neq 0$, we take $\epsilon_{1}=0$ and $\epsilon_{2}=\delta$, then equation (5.14) can be rewritten as

$$
\begin{aligned}
& \alpha_{\lambda} y_{2}=-\left(c_{11} y_{1}^{2}+c_{12} y_{1} y_{2}+c_{22} y_{2}^{2}\right)+o\left(|y|^{2}\right), \\
& \delta y_{1}=-\left(d_{11} y_{1}^{2}+d_{12} y_{1} y_{2}+d_{22} y_{2}^{2}\right)+o\left(|y|^{2}\right) .
\end{aligned}
$$

By the implicit function theorem, we obtain from (5.15) a function $y_{2}=f\left(y_{1}\right)$. Then (5.16) implies that

$$
\delta y_{1}+d_{11} y_{1}^{2}+d_{12} y_{1} f\left(y_{1}\right)+d_{22}\left(f\left(y_{1}\right)\right)^{2}+o\left(\left|y_{1}\right|^{2}\right)=0 .
$$


By the second-order nondegeneracy of $F_{\lambda}(x)$, there exist $a \neq 0$ and $k \geq 2$ such that (5.17) becomes

$$
\delta y_{1}+a y_{1}^{k}+o\left(\left|y_{1}\right|^{k}\right)=0 .
$$

We infer then from (5.18) that equations (5.15) and (5.16) have two solutions in $B_{r}$ : $(0,0)$ and $\left(\widetilde{y}_{1}, f\left(\widetilde{y}_{1}\right)\right)$, where

$$
\widetilde{y}_{1}=(-\delta / a)^{1 / k-1}+o(|\delta / a|),
$$

and $\delta$ is taken such that $\delta \cdot a<0$. Hence (5.14) has five solutions near $y=0$.

On the other hand, by Step 2, we know that (5.14), which can be expressed equivalently in the form of (5.2) and (5.1), has at most four solutions. This is a contradiction. Therefore, Assertion (3) is proved.

The proof of Theorem 5.1 is complete.

REMARK 5.2. The condition that $\left(a_{11}, a_{12}, a_{22}\right)$ and $\left(b_{11}, b_{12}, b_{22}\right)$ are linearly independent is necessary; otherwise Theorem 5.1 is not true.

6. An Application. Consider the following system of elliptic equations

$$
\left\{\begin{array}{l}
-\triangle u_{1}=\lambda u_{1}+a u_{2}+g_{1}\left(u_{1}, u_{2}\right) \\
-\triangle u_{2}=\lambda u_{2}+g_{2}\left(u_{1}, u_{2}\right)
\end{array}\right.
$$

supplemented with the Dirichlet boundary conditions

$$
u=\left(u_{1}, u_{2}\right)^{t}=0 \quad \text { on } \partial \Omega .
$$

Here the domain $\Omega \subset \mathbb{R}^{n}(n \leq 3)$ is a smooth and bounded domain, $\alpha_{1}, \alpha_{2}$ are real parameters, $a>0$ a constant, and $g_{1}, g_{2}$ smooth functions given by

$$
\begin{aligned}
& g_{1}=\sum_{i+j=2} a_{i j} u_{1}^{i} u_{2}^{j}+o\left(|u|^{2}\right), \\
& g_{2}=\sum_{i+j=2} b_{i j} u_{1}^{i} u_{2}^{j}+o\left(|u|^{2}\right) .
\end{aligned}
$$

Let $\lambda_{1}>0$ and $h_{1}$ be the first eigenvalue and eigenvector of the Laplacian operator with the Dirichlet boundary conditions:

$$
\begin{aligned}
& -\triangle h_{1}=\lambda h_{1}, \\
& \left.h_{1}\right|_{\partial \Omega}=0 .
\end{aligned}
$$

Let

$$
H=L^{2}\left(\Omega, \mathbb{R}^{2}\right), H_{1}=H_{0}^{2}\left(\Omega, \mathbb{R}^{2}\right),
$$

and let $L_{\lambda}=-A+B_{\lambda}=H_{1} \rightarrow H$, and $G=H_{2} \rightarrow H$ be defined by

$$
\begin{aligned}
& -A u=\left(\triangle u_{1}, \triangle u_{2}\right)^{t}, \\
& B_{\lambda}(u)=\left(\begin{array}{ll}
\lambda & a \\
0 & \lambda
\end{array}\right)\left(\begin{array}{l}
u_{1} \\
u_{2}
\end{array}\right), \\
& G(u)=\left(g_{1}(u), g_{2}(u)\right)^{t} .
\end{aligned}
$$


We consider two cases as follows.

Case $a=0$. In this case, we have $\beta_{1}(\lambda)=\beta_{2}(\lambda)=\lambda-\lambda_{1}$, and both the geometric and algebraic multiplicities are 2 . The corresponding eigenfunctions are given by

$$
e_{1}=e_{1}^{*}=\left(h_{1}, 0\right)^{t}, \quad e_{2}=e_{2}^{*}=\left(0, h_{1}\right)^{t} .
$$

Let $G_{1}$ be the 2 nd order homogeneous term of $G$ :

$$
G_{1}(u)=\left(\sum_{i+j=2} a_{i j} u_{1}^{i} u_{2}^{j}, \sum_{i+j=2} b_{i j} u_{1}^{i} u_{2}^{j}\right)^{t}
$$

Then by Theorem 3.1, if $x=\left(x_{1}, x_{2}\right)^{t}=0$ is an isolated singular point of

$$
\left\{\begin{array}{c}
\sum_{i+j=2} a_{i j} x_{1}^{i} x_{2}^{j}=0, \\
\sum_{i+j=2} b_{i j} x_{1}^{i} x_{2}^{j}=0,
\end{array}\right.
$$

then the problem (6.1) and (6.2) bifurcates at least one nontrivial branch from the trivial solution $u=0$ on each side of $\lambda=\lambda_{1}$. We remark here that the eigenvalue $\lambda=\lambda_{1}$ has even multiplicity 2, and the classical Krasnoselskii bifurcation theorem does not appear to be applicable.

Case $a \neq 0$ and $a_{11} \neq 0$. In this case, we have $\beta_{1}=\beta_{2}(\lambda)=\lambda-\lambda_{1}, \lambda_{0}=\lambda_{1}$, and

$$
\begin{aligned}
& e_{1}=\left(h_{1}, 0\right)^{t}, \quad e_{2}=\left(0, a^{-1} h_{1}\right)^{t}, \\
& L_{\lambda_{0}} e_{2}=e_{1}, \\
& e_{1}^{*}=\left(0, h_{1}\right)^{t}, \quad e_{2}^{*}=\left(a^{-1} h_{1}, 0\right)^{t}, \\
& L_{\lambda_{0}}^{*} e_{1}^{*}=e_{2}^{*} .
\end{aligned}
$$

Hence we have the geometric multiplicity $r=1$, and the algebraic multiplicity is still 2. The algebraic equations (3.7) become in this case

$$
a_{11} x^{2}=0 .
$$

When $a_{11} \neq 0, x=0$ is nondegenerate zero of (6.5). Namely, we obtain in this case, the problem (6.1) and (6.2) bifurcates at least one nontrivial branch from the trivial solution $u=0$ on each side of $\lambda=\lambda_{1}$. Again, as the eigenvalue $\lambda=\lambda_{1}$ has even algebraic multiplicity 2 , the classical Krasnoselskii bifurcation theorem does not appear to be applicable.

\section{REFERENCES}

[1] T. Kato, Perturbation theory for linear operators, Classics in Mathematics, Springer-Verlag, Berlin, 1995. Reprint of the 1980 edition.

[2] M. A. KrasnoseL'SKII, Topological methods in the theory of nonlinear integral equations, translated by A. H. Armstrong; translation edited by J. Burlak. A Pergamon Press Book, The Macmillan Co., New York, 1964.

[3] L. NiRenberg, Topics in nonlinear functional analysis, vol. 6 of Courant Lecture Notes in Mathematics, New York University Courant Institute of Mathematical Sciences, New York, 2001. Chapter 6 by E. Zehnder, Notes by R. A. Artino, Revised reprint of the 1974 original. 